\title{
Freckle Formation Affected by Geometry Features of Single Crystal Superalloy Castings
}

ISSN: 2576-8840

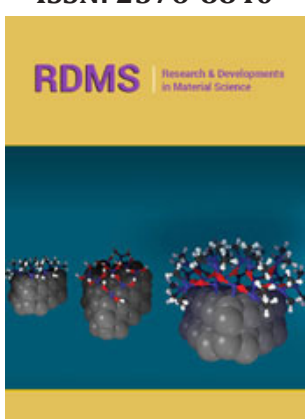

*Corresponding author: Dexin Ma Central South University, Changsha 410083, China

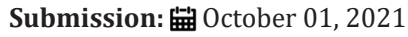

Published:

Volume 15 - Issue 5

How to cite this article: Dexin Ma*, Yunxing Zhao and Weitai Xu. Freckle Formation Affected by Geometry Features of Single Crystal Superalloy Castings. Res Dev Material Sci. 15(5). RDMS.000873. 2021. DOI: 10.31031/RDMS.2021.15.000873

Copyright@ Dexin Ma. This article is distributed under the terms of the Creative Commons Attribution 4.0 International License, which permits unrestricted use and redistribution provided that the original author and source are credited.

\author{
Dexin $\mathrm{Ma}^{1,2 *}$, Yunxing Zhao ${ }^{1,2}$ and Weitai $\mathrm{Xu}^{1}$ \\ ${ }^{1}$ Wedge Central South Research Institute, Shenzhen 518045, China \\ ${ }^{2}$ Central South University, Changsha 410083, China
}

\begin{abstract}
In comparison to our conventional knowledge, some new features of freckle appearance have been observed, indicating new aspects of freckle formation in the Single Crystal (SC) turbine blades made of superalloys. The casting shape has more significant influence on the freckle formation than the local thermal condition. On the transverse sections of the components with curved contour, freckles were exclusively found on the outward curving surfaces having positive curvature, because the surface effect zones of the neighboring sides are overlapped, providing more favorable convection condition. In comparison, the surfaces with negative curvature remained freckle free, because the surface effect of the neighboring sides is divergent from each other. In the longitudinal direction, the freckle formation can be promoted by contracting contour and suppressed by expanding one, respectively.
\end{abstract}

Keywords: Freckle; Superalloy; Single crystal; Geometry features

\section{Opinion}

Freckles are presently one of the main cast defects in the Single Crystal (SC) castings made of superalloys. It is now generally agreed that freckle occurrence is dependent on three factors: alloy chemistry, solidification conditions and casting size. For a defined alloy, thicker components are believed to be more freckle-prone, due to the lower solidification rate, large dendrite spacing and sufficient reservoir to support the interdendritic convection [1-5]. Besides the experimental investigations, a lot of studies have concentrated on the development of computational models and the Rayleigh-number-based criteria to describe the freckling onset [6-11]. However, the previous freckle studies were mostly based on examination of casting components with simple geometry, which were solidified under simplified conditions. The corresponding results are normally unsuitable for prediction and prevention of freckle formation in the industrial production of real SC castings.

In the recent works, a series of SC experiments using superalloy components with relatively complex geometry were carried out under industrial conditions, showing number of interesting phenomena about freckle formation. This will contribute to a better understanding of freckle formation in directionally solidified components of superalloys summarized as following new features: shadow-effect [11], step-effect [11,12], orientationeffect [13], slopping-effect [14], edge-effect [11,15].

In the present work, some SC turbine blades using second generation superalloys CMSX4 and DD5 (Table 1) were directionally solidified under industrial conditions. It was fund that the geometrical feature of castings plays a very important part in the freckle formation. Both of the geometry feature on transversal sections and the geometrical variation along the longitudinal direction can more effectively affect the freckle formation than the local thermal conditions. 
Table 1: Chemical compositions of the two superalloys used in the work(wt.\%).

\begin{tabular}{|c|c|c|c|c|c|c|c|c|c|c|}
\hline Alloy & Cr & Co & W & Mo & Al & Ti & Ta & Re & Hf & Ni \\
\hline CMSX-4 & 6.5 & 9.0 & 6.0 & 0.6 & 5.6 & 1.0 & 6.5 & 3.0 & 0.1 & Bal. \\
\hline DD5 & 7.0 & 7.5 & 5.0 & 1.5 & 6.2 & - & 6.5 & 3.0 & 0.15 & Bal. \\
\hline
\end{tabular}

Effect of transversal geometry feature (curvature effect)
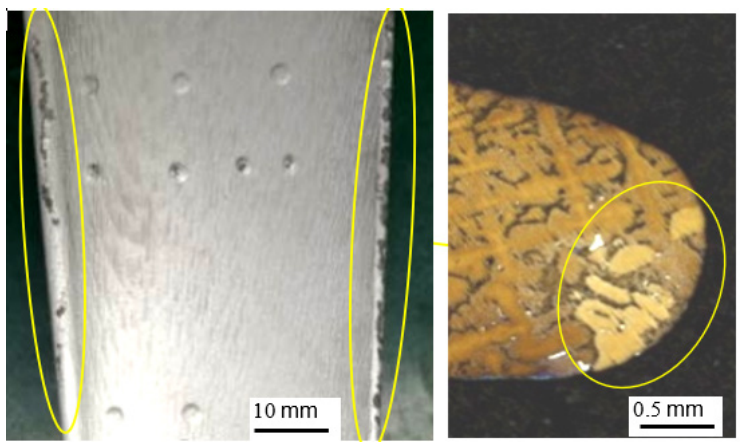

Figure 1: Freckles on the leading and trailing edge of a blade (a) and the corresponding cross section (b).

Figure 1 shows one of the produced turbine blades of alloy DD5, in which serious freckle defects occurred almost exclusively on the leading and trailing edges. This phenomenon cannot be explained with the current scientific knowledge. Ordinarily, the casting edges, especially the trailing edges of the turbine blades, are the thinnest portion and have the best cooling condition during solidification, where no freckles are expected. However, the observation in Figure 1 indicates clearly the promoting effect of the edge geometry on the freckle formation.

Figure 2 shows a shematic of the transversal section of a blade airfoil, in which the surface was cut into a closed curve. Four characteristic locations (A, B, C and D) are marked to indicate respectively the trailing and leading edge as well as the pressure and suction side of the airfoil. According to our experimental detection, the most serious freckle defect was observed on the trailing (A) and leading edge (B) having high curvature. Sometimes freckles were also observed on the position $C$ whose curvature is relatively low but still positive. On the position $\mathrm{D}$ with a negative curvature, however, no freckles have been detected. Therefore, the location ranking for the freckling tendency is A-B-C-D, just the same as their curvature ranking. This phenomenon was then called curvature effect of freckle formation. This appears to contradict the current knowledge about the effect of size and thermal condition, which gives the exact opposite prediction for the freckling tendency ranking in Figure 2.

Freckles are a surface defect whose formation is promoted by the surface contacting with the mold wall, revealing the so called surface effect or wall effect. In our previous study [16], the flow permeability near the casting surface was examined to be about one order of magnitude higher than that inside the mushy zone. A boundary layer was defined to indicate the depth of surface effect. At the convex edges having positive curvature, such as at site A, B, and $C$ in Figure 2, the surface effect zones of the neighboring sides are overlapped, providing more favorable freckling condition. On the concave edge having negative curvature, such as location D in Figure 2, the surface effect of the neighboring sides is divergent from each other, resulting normally a freckle free zone. This can be called as the curvature effect of the transversal geometry on the freckle formation, corresponding to the edge effect in the polygonal sections of castings [11]. In hollow blades, the occurrence of the internal freckles is strongly influenced by the curvature of the inserted ceramic cores.

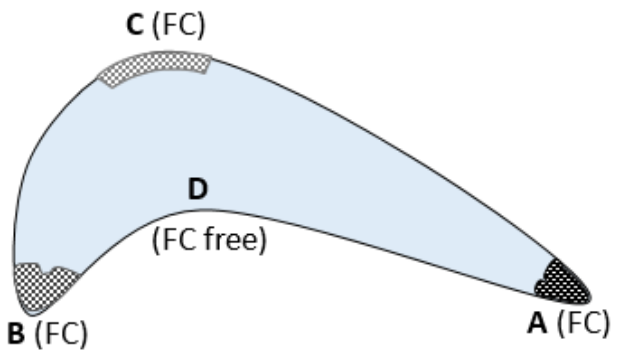

Figure 2: Schematic of the transversal contour of a blade showing the freckling (FC) locations.

\section{Effect of longitudinal geometry feature (contracting and expanding effect)}
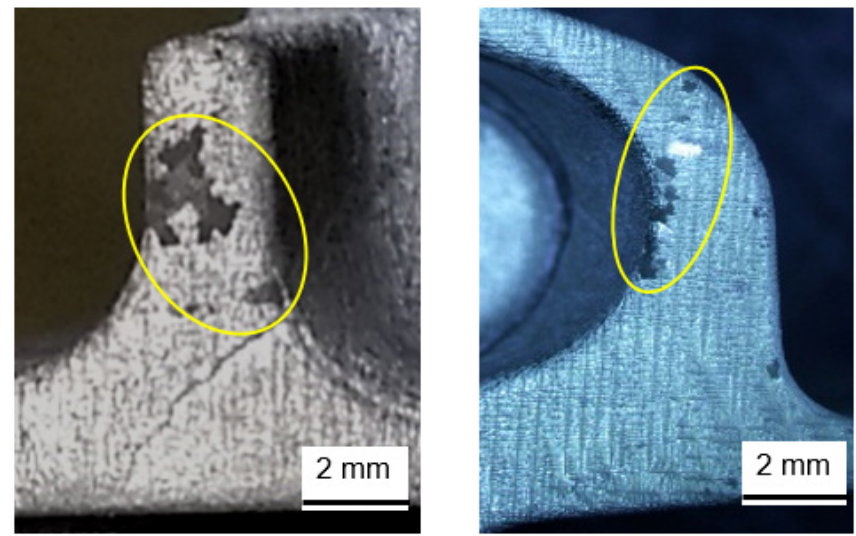

Figure 3: Two examples of freckle formation induced by the vertical geometry contraction in the blades of alloy DD5.

According to the well-known effect of casting size and thermal condition, the castings should become less freckle prone as their thickness decreases, because of the increase in the local cooling rate. In Figure 3, however, the opposite phenomenon was observed. The solidification of turbine blades was performed vertically, revealing a geometrical ruduction in solidification direction. The freckles did not occurr on the thicker portions at the low levels, but at the narrow passes after a geometrical contraction, just in contrary to our current knowledge.

Figure 4 shows a SC blade of alloy CMSX-4 with freckles in some characteristic positions. The blade was vertically solidified from bottom to top. At position A, the contour of the blade is reduced, leading to the corresponding occurrence of freckles. At the 
subquent enlargement of the contour at $\mathrm{B}$, the existing freckle chain disapeared, instead of further growth. Position $C$ is the transition from platform into airfoil, acompanied with a significant reduction in contour. As a result a freckle chain was formed on the edge and developed further along the edge. Position D is the geometrical transition from airfoil to the upper shroud. Due to the geometrical enlargement the existing freckle chain was stopped to grow and the solidification structure became freckle free again.
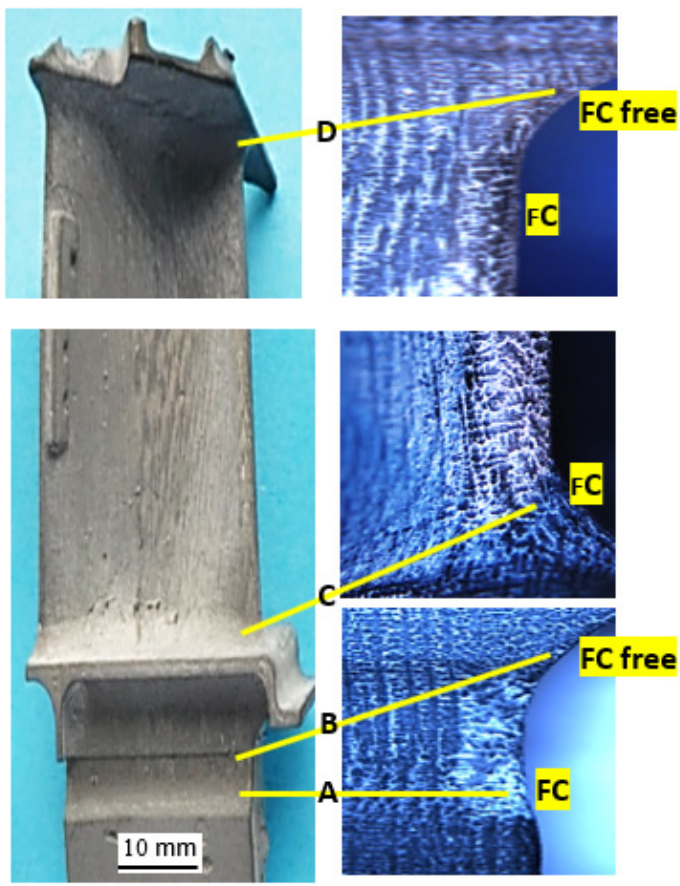

Figure 4: Freckle (FC) formation affected by vertical geometry variation in a SC blade, revealing both the promoting and suppressing effect of the geometrical contracting (A and $\mathrm{C}$ ) and expanding (B and $\mathrm{D}$ ), respectively.

According to the well-known size effect on freckle formation, the freckling extent becomes more pronounced as the casting size increases. However, in Figure 4 the contrary phenomena were observed. All of the contracting portions in the investigated blade exhibit serious freckles. In contract to this, no freckles were observed on the surfaces of any portion possessing a size enlargement in the solidification direction.

The mechanism for how the longitudinal variation of casting geometry affects the freckle formation is not very clear. It is believed that the contracting geometry in solidification direction means higher surface/volume ratio and more surface effect. Moreover, this geometry feature provides a wider supporting reservoir from below for freckling flow in the upper narrow pass. In the case of size enlargement in solidification direction, the surface/volume ratio becomes lower, leading to an insufficient surface effect for the freckle formation. Moreover, the geometrical lack of the convection reservoir from below becomes another factor to suppress the freckle formation.

\section{Development of freckles into stray grains}

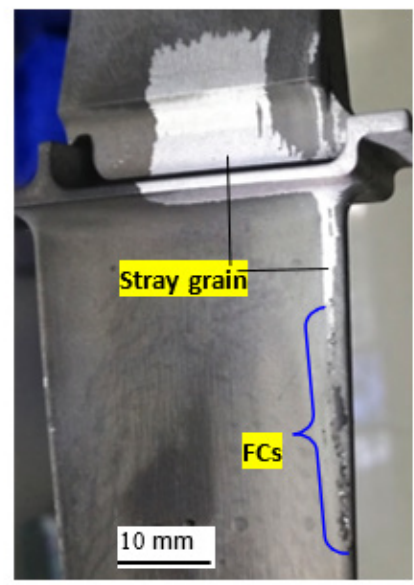

(a)

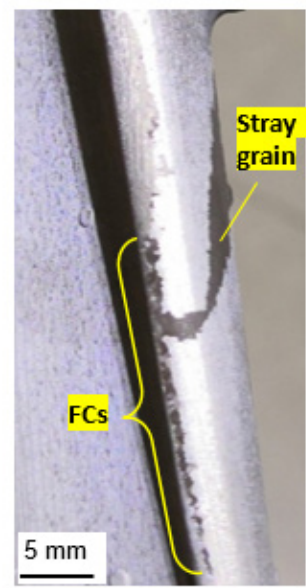

(b)
Figure 5: Stray grains originated from the freckles (FC) on the blade edges of alloy DD5.

As stated above, the freckles are often observed on the leading and trailing edges due to the high surface curvature. During solidification, the sharp edges have the best cooling condition in the blade, resulting in a faster dendrite growth ahead of the neighboring suction and pressure sides. In the advanced mushy zone on the blade edges, the small equiaxed grains forming the freckle chain can easily grow vertically ahead (Figure 5a) or laterally into the blade sides (Figure 5b). In these cases, the freckles characterized as chain-like surface defect developed finally into stray grains, a 3-dimensional structure defect of large size. This phenomena is not often observed in the components of simple geometry, such as cylindrical bars, in which a much flatter solidification front could be established.

In summary, freckle formation in SC castings was fund to be very strongly effected by their geometrical feature, so that the influence of local thermal condition could be neglected. On the transverse sections of the castings with curved contour, freckles were exclusively found on the outward curving surfaces having positive curvature, whereas the surfaces with negative one remained freckle free. In the longitudinal direction, the freckle formation can be promoted by contracting contour and suppressed by expanding one, respectively. The freckles on the casting edges can laterally or longitudinally develop into the stray grains, becoming another typical defect in SC castings.

\section{References}

1. Giamei AF, Kear BH (1970) On the nature of freckles in nickel base superalloys. Metall Trans 1: 2185-2192.

2. Copley SM, Giamei AF, Johnson SM (1970) The origin of freckles in unidirectional solidification. Metall Trans 1: 2193-2203

3. Auburtin P, Wang T, Cockcroft SL (2000) Freckle formation and freckle criterion in superalloy castings. Metall Mater Trans 31B: 801-811.

4. Tin S, Pollock TM (2004) Predicting freckle formation in single crystal Ni-base superalloys. J Materials Science 39: 7199-7205. 
5. Auburtin P (1998) Determination of the influence of growth fron angle on the freckle formation in superalloys. PhD Thesis, Uni of British Columbia, Canada.

6. Auburtin P, Cockcroft SL, Mitchella (1996) Liquid density inversion during the solidification of superalloys and their relationship to freckle formation in castings. Superalloys 1996, Kissinger RD (Ed.), TMS, 1996: 443-450

7. Beckmann C, Gu JP, Boettinger WJ (2003) Development of a freckle predictor via Rayleigh number method for single-crystal Ni-base superalloy castings. Metall Mater Trans 31A: 2545-2557.

8. Yang W, Chen W, Chang KM (2001) Freckle criteria for the upward directional solidification of alloys. Metall Mater Trans 32A: 397-406.

9. Tewari SN, Tiwari R, Magadi G (2004) Mushy-zone Rayleigh number to describe macrosegregation and channel segregate formation during directional solidification of metallic alloys. Metall Mater Trans 35A: 2927-2934.

10. Feng Q, Carroll LJ, Pollock TM (2006) Solidification segregation in ruthenium-containing nickel-base superalloys. Metall Mater Trans 37A: 1949-1962.
11. Ma D, Wu Q, Bührig-Polaczek A (2012) Some new observations on freckle formation in directionally solidified superalloy components. Metall Mater Trans 43B: 344-353.

12. Ma D, Ziehm J, Wang W, Bührig-Polaczek A (2011) Freckle formation in directionally solidified superalloy components with expanding crosssection. IOP Conf Series: Mater Sci Eng 27: 012034.

13. Ma D, Mathes M, Zhou B, Bührig-Polaczek A (2011) Influence of crystal orientation on the freckle formation in directionally solidified superalloys. Adv Mater Res 278: 114-119.

14. Ma D, Bührig-Polaczek A (2014) The geometry effect on freckle formation in the directionally solidified superalloy CMSX-4. Metall Mater Trans 45A: 1435-1444

15. Ma D, Dong Z, Wang F, Dong H (2020) A phenomenological analysis of freckling in directional solidification of Ni-base superalloys: the role of edge and curvature in casting components. Metall Mater Trans A 51A(1): 88-92.

16. Ma D, Sahm PR (1996) Forced decanting of solidification front of a technical Al-Si alloy. Aluminium 72: 905-909.

For possible submissions Click below: 